\title{
Finite Element Analysis of MHD Natural Convection in a Rectangular Cavity and Partially Heated Wall
}

\author{
Md. Shahidul Alam ${ }^{1}$, Md. Shirazul Hoque Mollah ${ }^{1}$, Md. Abdul Alim², Md. Kazi Humayun Kabir ${ }^{3}$ \\ ${ }^{1}$ Department of Mathematics, Dhaka University of Engineering and Technology, (DUET), Gazipur, Bangladesh \\ ${ }^{2}$ Department of Mathematics, Bangladesh University of Engineering and Technology, (BUET), Dhaka, Bangladesh \\ ${ }^{3}$ Department of Mathematics, Mohammadpur Kendriya College (MKC), Dhaka, Bangladesh
}

Email address:

shdlalam@gmail.com (Md. S. Alam),a0alim@gmail.com (Md. A. Alim), mollah123@ yahoo.com (Md. S. H. Mollah), kfzkabir@gmail.com (Md. K. H. Kabir)

\section{To cite this article:}

Md. Shahidul Alam, Md. Shirazul Hoque Mollah, Md. Abdul Alim, Md. Kazi Humayun Kabir. Finite Element Analysis of MHD Natural Convection in a Rectangular Cavity and Partially Heated Wall. Engineering and Applied Sciences. Vol. 2, No. 3, 2017, pp. 53-58. doi: $10.11648 /$ j.eas. 20170203.12

Received: May 30, 2017; Accepted: June 16, 2017; Published: July 18, 2017

\begin{abstract}
In this paper numerical study is presented of two-dimensional laminar steady-state on megneto-hydrodynamics (MHD) free convection for heat flow patterns within rectangular enclosures. A finite element analysis is performed to investigate the effects of uniform heating and is also used for solving the Navier-Stokes and Energy balance equations. The horizontal bottom wall is divided into three sections. The middle section of the horizontal bottom wall was kept temperature at $\mathrm{T}_{\mathrm{h}}$ and the other two parts of the horizontal bottom wall were kept thermal insulation while the left and right vertical walls and the top wall of the cavity were maintained constant temperature $T c$ with $T_{h}>T_{c}$. Parametric studies of the fluid flow and heat transfer in the enclosure are performed for magnetic parameter Hartmann number $(\mathrm{Ha}=0,50,100)$, Rayleigh number $(\mathrm{Ra}=$ $10^{3}-10^{6}$ ) and Prandtl number $\operatorname{Pr}=0.71$. The streamlines, isotherms, average Nusselt number at the hot wall and velocity profiles and temperature distribution of the fluid in the enclosure are presented for the parameters. The numerical results indicated that the Hartmann number and Rayleigh number have strong influence on the streamlines and isotherms. Also the mentioned parameters have significant effect on average Nusselt number at the hot wall and average temperature of the fluid in the enclosure.
\end{abstract}

Keywords: Free Convection, Magnetic Field, Rectangle Cavity, Numerical Solution

\section{Introduction}

The basic problem of free convection in cavity has received considerable attention from researchers. Most of the cavities commonly used in industries are cylindrical, rectangular, square and triangular etc. Rectangular cavities have received a more considerable attention for its application in various fields. Tanmay et al. [1] studied mixed convection flow within a square cavity with uniform and non-uniform heating of bottom wall. Reddy [2] introduced

Finite element analysis to describe the discretized energy and momentum equations subjected to the boundary conditions simultaneously. Lyican and Bayazitoglu [3] performed an analytical study of natural convective heat transfer within trapezoidal enclosure. Roy and Basak [4] studied Finite element analysis of natural convection flows in a square cavity with non-uniformly heated wall(s). Kuyper and Hoogendoorn [5] investigated Laminar natural convection flow in trapezoidal enclosures to study the influence of the inclination angle on the flow and the dependence of the average Nusselt numbers on the Rayleigh number. Varol et al. [6, 7] studied magnetohydrodynamic (MHD) for various inclinations of trapezoidal enclosures filled with either fluid or porous medium on natural convection. Oztop et al. [8] investigated natural convection in wavy enclosures withvolumetric heat sources. Kahveci and Öztuna [9] studied MHD natural convection flow and heat transfer in a laterally heated partitioned enclosure. Basak et al. [10] investigated energy flows due to natural convection within trapezoidal enclosures with hot bottom wall and cold side walls in the presence of insulated top walls. Basak et al.[10] investigated energy flows due to natural convection 
within trapezoidal enclosures with hot bottom wall and cold side walls in the presence of insulated top walls. Molla et al. [11] have studied problem of magnetohydrodynamic natural convection flow on a sphere in presence of heat generation or absorption. Nithyadevi et al [12] using a numerical simulation on magnetohydrodynamic natural convection in a square cavity with partially heated cooled side walls. They found that the flow and the heat transfer rate in the cavity affected by the sinusoidal temperature profile and by the magnetic field at lower values of Grashof number. Moreover they found that the maximum rate of heat transfer occurs for the active portions located at the middle of the side walls. Mahmoodi and Taleapour [13] investigated numerically magnetohydrodynamic free convection in a square cavity with hot left wall, cold top wall and insulated right and bottom wall. They found that a clockwise primary eddy inside the cavity regardless the Rayleigh number and Hartman number. Also they found that the magnetic field decrease the intensity of free convection and flow velocity. Recently Hasanuzzaman et al. [14] investigated Magnetohydrodynamic natural convection in trapezoidal cavities. Mustafizur Rahman et al. [15] investigated unsteady mixed convection in a porous media filled lid-driven cavity heated by semi-circular heaters. They found that multiple circulation cells are formed for the lowest value of Darcy number due to domination of natural convection heat transfer. Heat transfer decreased with decreasing of Darcy number and Richardson number. Flow strength increases with increasing of dimensionless time increment. Semicircular heaters make small effects on mixed convection flow and heat transfer due to small diameters. K. Md. Rabbia [16] Numerical investigation of pure mixed convection in a ferro fluid-filled lid-driven cavity for different heater configurations. O. S. K. Hamama, et. al. [17] conducted irreversibility investigation on MHD natural convection in a square cavity for different Prandtl numbers. The findings of them are the magnetic field parameter suppresses the flow and this leads to a decrease of entropy generation. Temperature decreases with the increase of the magnetic field parameter. The average Nusselt number increases with the Prandtl number and, in particular, its effect is more evident for higher values of Hartmann number. B. C. Shekar and N. Kishan [18], Finite element analysis of natural convective heat transfer in a porous square cavity filled with nanofuids in the presence of thermal radiation. Shahidul Alam1 et.al. [18] Studied numerical simulation of natural convection in a rectangular cavity with corner heater in presence of magnetic field.

In the light of the above literature review and to the best of authors' knowledge no article has yet been published which have addressed natural convection in a rectangular cavity in presence of magnetic field with partially heated wall. So, the purpose of the present study is to investigate the effects of MHD on natural convection in a rectangular cavity and partially heated walls numerically. The results are presented in terms of streamlines and isotherms inside the cavity, vertical component of the velocity along the horizontal centerline of the cavity, local Nusselt number along the hot wall, and average Nusselt number of the hot wall.

\section{Physical Model}

Geometry of the rectangular cavity with boundary conditions considered in the present paper is shown in Figure 1. The height and length of the cavity are denoted $\mathrm{H}$ and $\mathrm{L}$ respectively. The middle section of the horizontal bottom wall was kept temperature at $T_{h}$ and the other two parts of the horizontal bottom wall were kept thermal insulation while the left and right side and the top walls of the cavity were maintained constant temperature Tc with $T_{h}>T c$. The length of the cavity perpendicular to its plane is assumed to be enough; hence the problem is considered two dimensional. The magnetic field $\mathrm{B} 0$ is applied parallel to $\mathrm{x}$-axis. The cavity is filled with an electric conductivity fluid with $\mathrm{pr}=$ 0.71 that is considered Newtonian and incompressible. The fluid flow is assumed to be laminar. The thermo physical properties of the fluid are considered constant with the exception of the density which varies according to the Boussinesq approximation..

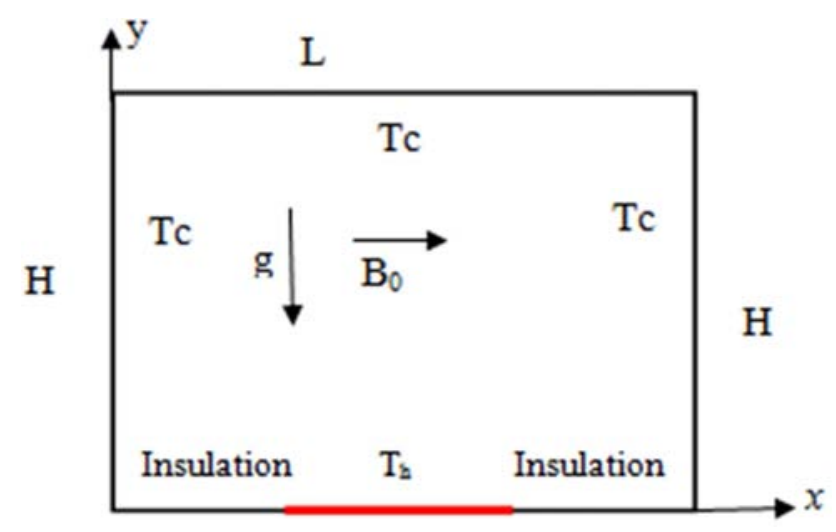

Figure 1. Schematic view of the cavity with boundary conditions considered in the present paper.

\section{Mathematical Formulation}

The continuity, momentum and energy equations for laminar, steady state, two-dimensional free convection with a magnetic field in $\mathrm{x}$-direction, in non-dimension are as follows:

$$
\begin{gathered}
\frac{\partial U}{\partial X}+\frac{\partial V}{\partial Y}=0 \\
U \frac{\partial U}{\partial X}+V \frac{\partial U}{\partial Y}=-\frac{\partial P}{\partial X}+\operatorname{Pr}\left(\frac{\partial^{2} U}{\partial X^{2}}+\frac{\partial^{2} U}{\partial Y^{2}}\right) \\
\rho\left(\frac{\partial V}{\partial X}+\frac{\partial V}{\partial Y}\right)=-\frac{\partial P}{\partial Y}+\mu\left(\frac{\partial^{2} V}{\partial X^{2}}+\frac{\partial^{2} V}{\partial Y^{2}}\right)+R a \operatorname{Pr} \theta-H a^{2} \operatorname{Pr} V
\end{gathered}
$$




$$
U \frac{\partial \theta}{\partial X}+V \frac{\partial \theta}{\partial Y}=\left(\frac{\partial^{2} \theta}{\partial X^{2}}+\frac{\partial^{2} \theta}{\partial Y^{2}}\right)
$$

Where $\mathrm{Ra}, \mathrm{Pr}$ and $\mathrm{Ha}$ are the Rayleigh, Prandtl and Hartman numbers and are defined as:

$$
R a=\frac{g \beta\left(T_{h}-T_{c}\right) L^{3}}{\alpha v}, \operatorname{Pr}=\frac{v}{\alpha}, H a=B_{0} L \sqrt{\frac{\sigma}{\rho v}}
$$

The effect of magnetic field into the momentum equation is introduced through the Lorentz force term $\bar{J} \times \bar{B}$ that is reduced to $-\sigma_{0} B_{0} v^{2}$

To computation of the rate of heat transfer, Nusselt number along the hot wall of the enclosure is used that is as follows:

$$
N u=\frac{1}{L} \int_{0}^{L} N u_{\text {local }} d x
$$

The boundary conditions are:

On the left wall of the rectangular cavity:

Present work
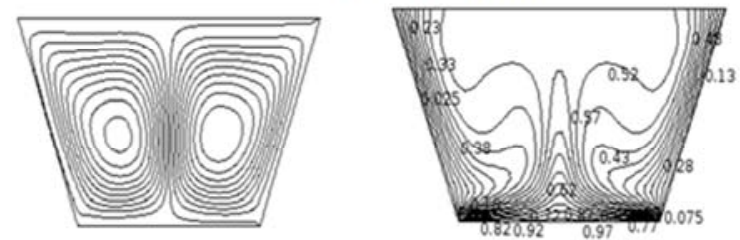

$U=0, V=0, \theta=0$ and right wall of the cavity:

$U=0, V=0, \theta=0$

On the heated block: $U=0, V=0, \theta=1$

\section{Numerical Advance}

Partial differential equations governing the flow and temperature field are solved by using finite element method. The quadratic triangular element is used to develop the finite element equations. For the velocities and temperature all the six nodes are used and for the pressure only the corner nodes are used. Different types of grid densities have been selected to assess the accuracy of the numerical simulation procedure. The nonlinear algebraic equations arising from the finite element formulation are solved by applying the NewtonRaphson iteration technique. Validation of the code was done by comparing streamlines and isotherms with results shown in Fig. 2 by Hasanuzzaman et al. [15]. As can be observed from the figure, very good agreement exists between the two results

\section{Hasanuzzaman et al.}
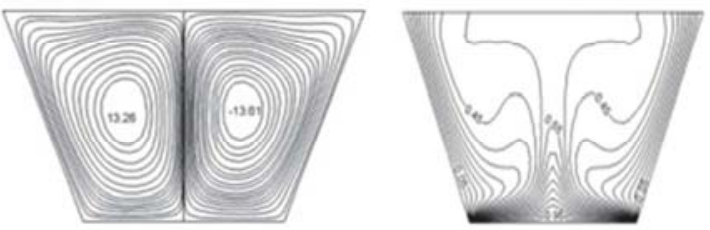

Figure 2. Comparison of streamlines and isotherms for $\mathrm{Ha}=10$ and $\mathrm{Ra}=10^{6}$.

\section{Result and Discussion}

In this section, results of the numerical study on magnetohydrodynamic free convection fluid flow and heat transfer in a rectangle cavity field with an electric conductive fluid with $\operatorname{Pr}=0.71$ are presented. The results have been obtained for the Rayleigh number ranging from $10^{3}$ to $10^{6}$ and the Hartman number varying from 0 to 100 . The results are presented in terms of streamlines and isotherms inside the cavity, the vertical velocity component along the horizontal midline of the cavity, the local Nusselt number along the hot wall. Variation of streamlines and isotherms inside the cavity with Rayleigh number and Hartman number are shown in Fig. 3 and 4 respectively. As can be observed from the figures with existence of symmetrical boundary conditions about the vertical centerline of the cavity, the flow and temperature fields are symmetrical about this line. As can be seen from the streamlines in the Fig. 3, a pair of counterrotating eddies are formed in the left and right half of the cavity for all Rayleigh number and Hartman numbers considered. Each cell ascends through the symmetry axis, then faces the upper wall and moves horizontally and finally descends along the corresponding cold side wall. Condition dominant heat transfer is observed from the isotherms in Fig. 4 at $\mathrm{Ra}=10^{3}$ and $\mathrm{Ha}=0$. With increase in Rayleigh number, the isotherms are condensed next to the side walls which mean increasing heat transfer through convection. Formation of thermal boundary layers can be found from the isotherms at $\mathrm{Ra}=10^{5}$ and $10^{6}$ and at $\mathrm{Ha}=0$. At $\mathrm{Ha}=100$ the shape of the core of eddies converts to isosceles triangle and locates close to the hot bottom wall.

Variation of the vertical velocity component along the horizontal centerline of the cavity with the Rayleigh number and for $\mathrm{Ha}=0$ are shown in Fig. 5(a). It can be seen from the figure that the absolute value of maximum and minimum value of velocity increases with increasing the Rayleigh number (increasing the buoyant force). Effect of Hartman number on vertical component of the velocity along the horizontal midline of the cavity at $\mathrm{Ra}=10^{6}$ is shown in Fig. 5(b). As can be seen from the figure with increase in Hartman number motivates the flow velocity to decreases. A very slow fluid velocity occurs at $\mathrm{Ha}=100$. It is found that free convection heat transfer decreases with increase in fluid velocity via increasing the Hartman number. At $\mathrm{Ha}=100$ the shape of the core of eddies converts to isosceles triangle and locates close to the hot bottom wall. Variation of the vertical velocity component along the horizontal centerline of the cavity with the Rayleigh number and for $\mathrm{Ha}=0$ are shown in Fig. 5a. It can be seen from the figure that the absolute value of maximum and minimum value of velocity increases with increasing the Rayleigh number (increasing the buoyant 
force). Effect of Hartman number on vertical component of the velocity along the horizontal midline of the cavity at $\mathrm{Ra}=$ $10^{6}$ is shown in Fig. 5b. As can be seen from the figure with increase in Hartman number motivates the flow velocity to
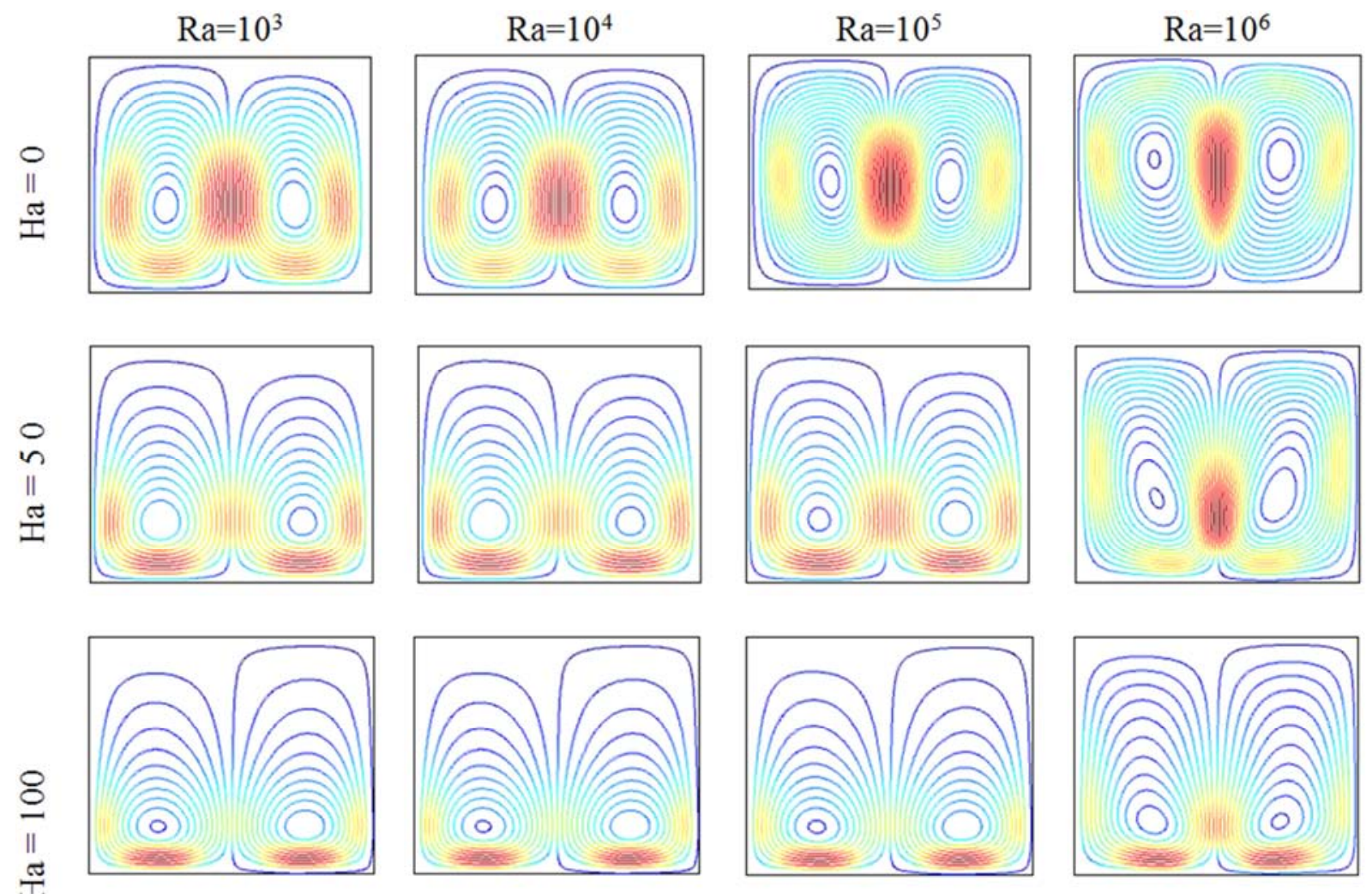

Figure 3. Streamlines at different Rayleigh numbers and Hartman numbers.
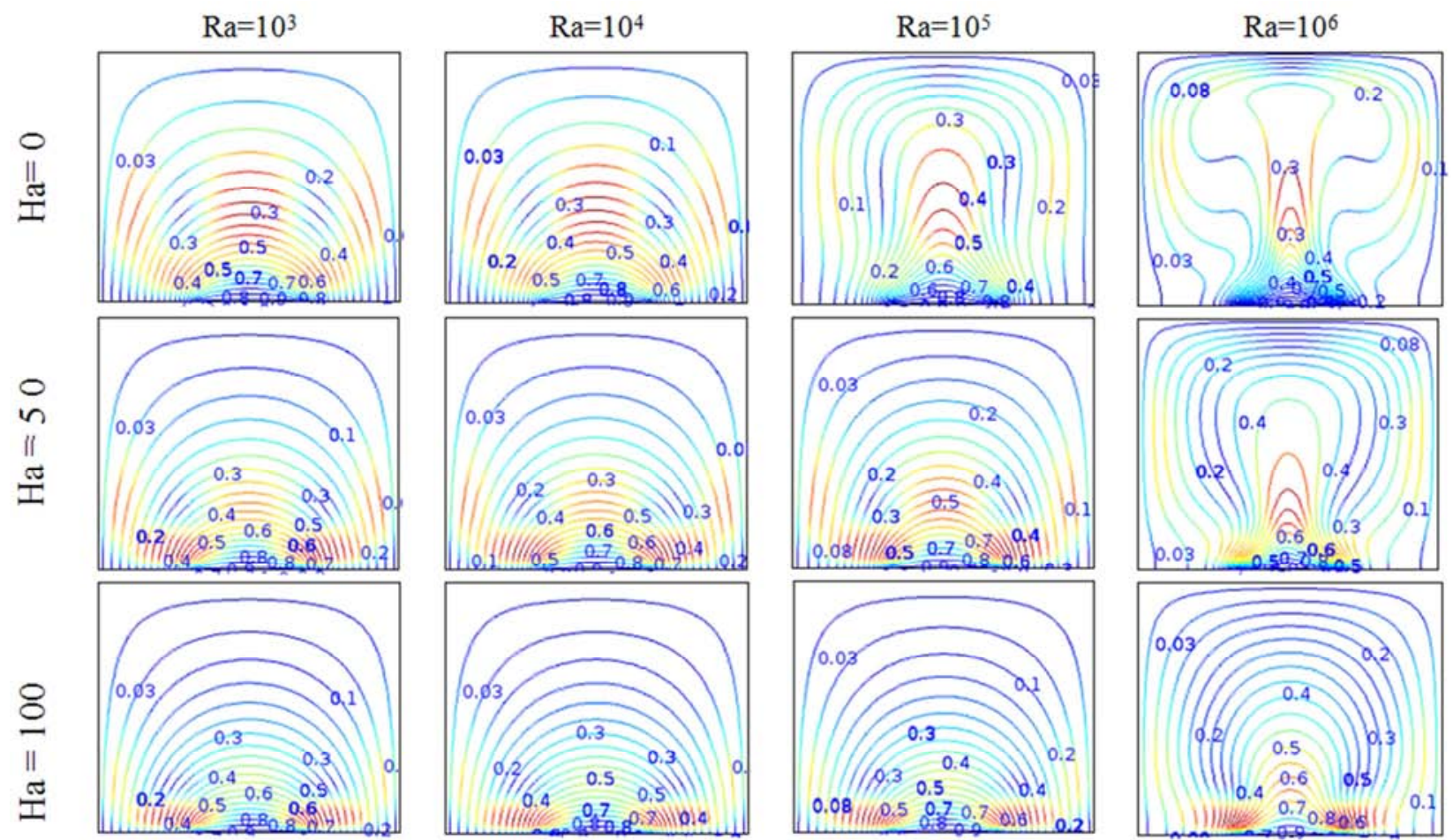

Figure 4. Isotherms at different Rayleigh numbers and Hartman numbers. 


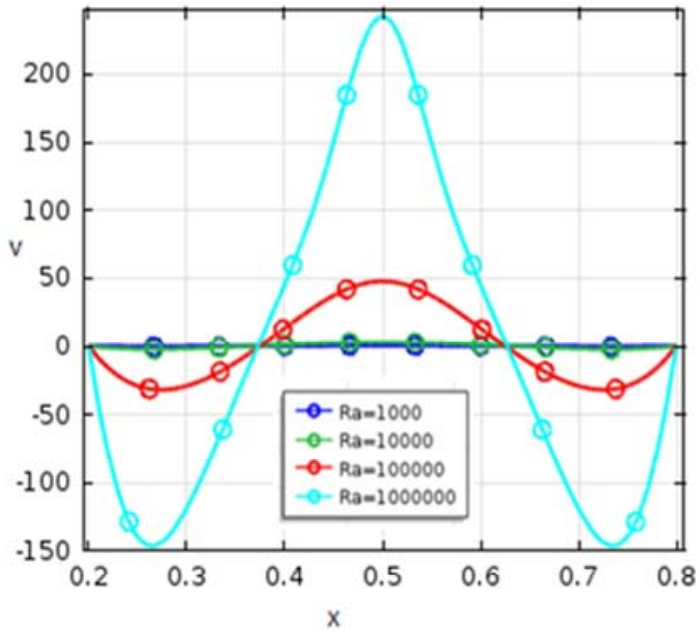

(a)

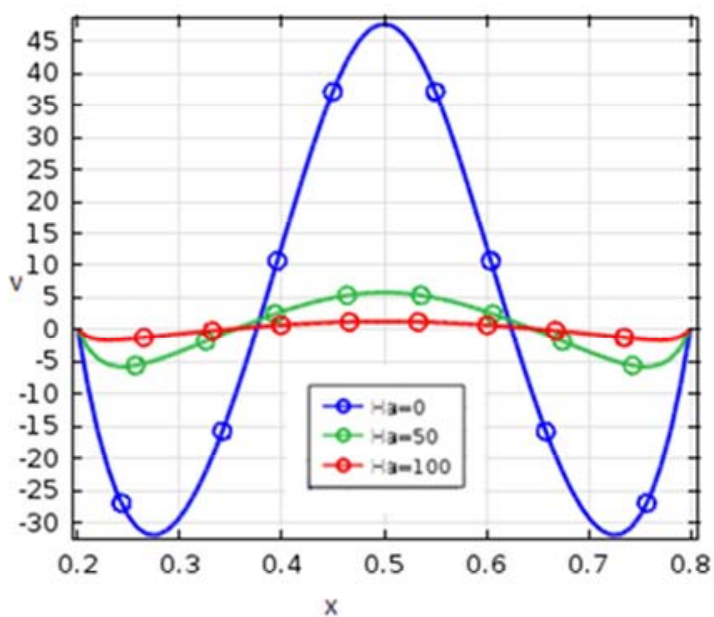

(b)

Figure 5. Variation of vertical velocity component along the horizontal central line of the cavity (a) with Rayleigh number at Ha $=0$. (b) With Hartman number at $R a=10^{6}$.

\section{Conclusion}

Using a numerical simulation based on the finite element method, the Magnetohydrodynamic free convection fluid flow and heat transfer in a rectangle cavity heated from below and cooled from vertical walls filled with an electric conductive fluid with Prandtl number of 0.71 was studied numerically. The numerical procedure was validated by comparing the average Nusselt number for a differentiallyheated rectangle enclosure obtained by the code with the existing results in the literature. Very good agreements were observed between them. Subsequently, a parametric study was performed and the effects of Rayleigh number and the Hartman number on the fluid flow and heat transfer were investigated.

For all cases considered, two counter rotating eddies were formed inside the cavity regardless the Rayleigh and the Hartman number. The obtained result showed that the heat transfer mechanism, temperature distribution and the flow characteristics inside the cavity depended strongly upon both the strength of the magnetic field and the Rayleigh number. Also it was found that using the longitudinal magnetic field results in a force (Lorentz force) opposite to the flow direction that tends to decrease the flow velocity, Moreover it was observed that, for low Rayleigh number, by increase in the Hartman number, free convection is suppressed and heat transfer occurs through conduction mainly.

\section{References}

[1] TanmayBasak, S. Roy, PawanKumer Sharma and I. Pop, Analysis of mixed convection flow within a square cavity with uniform and non-uniform heating of bottom wall, International Journal of Thermal Science, Vol. 48, pp. 891912, 2009.

[2] J. N. Reddy, An introduction to Finite element method, McGraw-Hill, New York, 1993.
[3] L. Lyican and Y. Bayazitoglu, An analytical study of natural convective heat transfer within trapezoidal enclosure, ASME Trans. J. Heat transfer, Vol. 102, pp. 640-647, 1980.

[4] S. Roy and T. Basak, Finite element analysis of natural convection flows in a square cavity with non- uniformly heated wall(s), Int. J. Engrg. Science, Vol. 45, pp. 668-680, 2005.

[5] R. A. Kuyper and C. J. Hoogendoorn, Laminar natural convection flows in trapezoidal enclosures, Num. Heat transfer, A Vol. 28, pp. $55-67,1995$.

[6] Y. Varol, H. F. Oztop and I. Pop, Numerical analysis of natural convection in an inclined trapezoidal enclosure filled with a porous medium, Int. J. Thermal Science, Vol. 47, No.10, pp. 1316-1331, 2008

[7] Y. Varol, H. F. Oztop and I. Pop, natural convection in rightangle porous trapezoidal enclosure with partially cooled from inclined wall. International Communications in Heat and Mass Transfer, Vol. 36, No. 1, pp. 6-15, 2009.

[8] Hakan F. Oztop, Eiyad Abu-Nada, Yasin Varoland Ali Chamkha, Natural convection in wavy enclosures with volumetric heat sources, Int. J. Thermal Science, Vol. 50, pp. 502-514, 2011

[9] Kamil Kahveci and Semiha Öztuna, MHD natural convection flow and heat transfer in a laterally heated partitioned enclosure, European Journal of Mechanics - B/Fluids Vol. 28(6), pp. 744-752, 2009.

[10] Tanmay Basak, S. Roy and I. Pop, Heat flow analysis for natural convection within trapezoidal enclosures based on heatline concept, Int. J. Heat Mass Transfer, Vol. 52, pp. 24712483, 2009.

[11] Md. M. Molla, M. A. Taher, Md. M. K. Chowdhury and Md. A. Hossain, Magnetohydrodynamic Natural Convection Flow on a sphere in Presence of Heat Generation, Nonlinear Analysis: Modelling and Control, Vol. 10, No. 4, pp. 349-363, 2005.

[12] Nithyadevi, P. Kandaswamy, S. MalligaSundari, "Magnetoconvtion in a square cavity with partially active vertical walls: Time periodic Boundary condition," Int. J. Heat Mass Tran., 52, 2009, pp. 1945-195. 
[13] M. Mahmoodi, Z. Talea'pour, "Magnetohydrodynamic Free Convection Heat Transfer in a Square Enclosure heated from side and cooled from the ceiling," Computational Thermal Sciences, vol. 3, 2011, 219-226.

[14] M. Hasanuzzaman, Hakan F. Öztop, M. M. Rahman, N. A. Rahim, R. Saidur, Y. Varol, “ Magnetohydrodynamic natural convection in trapezoidal cavities," International Communications in Heat and Mass Transfer 39, 1384-1394, 2012.

[15] M. M. Rahman, H. F. Oztop, R. Saidur, S. mekhilef and K. AlSalem "Unsteady mixed convection in a porous media filled lid-driven cavity heated by a semi-circular heaters," Thermal Science, Vol. 19, No. 5, pp. 1761-1768 Year 2015.
[16] K. M. Rabbia, S. Sahaa, S. Mojumdera, M. M. Rahmanb, R. Saidurc and T. A. Ibrahimd Numerical investigation of pure mixed convection in a ferrofluid-filled lid-driven cavity for different heater configurations, Alexandria Engineering Journal, Volume 55, Issue 1, Pages 127-139, 2016.

[17] O. S. K. Hamama, F. S. Sharif, M. N. Hidouri and T. alAqqad, Irreversibility investigation on MHD natural convection in a square cavity for different Prandtl numbers, African Journal of Physics Vol. 3 (4), pp. 090-104, 2016.

[18] Md. Shahidul Alam, Md. S. H. Mollah, A. H. Bhuiyan and M. A. Alim, Numerical Simulation of Natural Convection in a Rectangular Cavity with Corner Heater in Presence of Magnetic Field, Journal of Computer and Mathematical Sciences, Vol. 8(6), 215-225 2017. 